\title{
PERIODIC AND HOMOGENEOUS STATES ON A VON NEUMANN ALGEBRA. II $^{1}$
}

\author{
BY MASAMICHI TAKESAKI \\ Communicated by Jacob E. Feldman, August 15, 1972.
}

This paper is a natural continuation of the previous paper [9]. In [9], we proved a structure theorem for a von Neumann algebra with a fixed periodic and homogeneous state. In this paper, we will show that the structure theorem in [9] determines intrinsically the algebraic type of a factor with a periodic and inner homogeneous state (see Definition 1). We keep the terminologies and the notations in [9].

DEFINITION 1. A normal state $\varphi$ on a von Neumann algebra $\mathscr{M}$ is said to be inner homogeneous if $G(\varphi) \cap \operatorname{Int}(\mathscr{M})$ acts ergodically on $\mathscr{M}$, that is, if the group of all inner automorphisms of $\mathscr{M}$ leaving $\varphi$ invariant has no fixed points other than the scalar multiples of the identity.

For each $a \in \mathscr{M}$, we write

$$
\operatorname{Ad}(a) x=a x a^{*}, \quad x \in \mathscr{M} .
$$

Since $\operatorname{Ad}(u) \in G(\varphi)$ for a unitary $u \in \mathscr{M}$ if and only if $u$ falls in $\mathscr{M}_{\varphi}$, the centralizer of $\varphi$, the inner homogeneity of $\varphi$ is equivalent to the fact that $\mathscr{M}_{\varphi}^{\prime} \cap \mathscr{M}=\{\lambda 1\}$. Hence $\mathscr{M}_{\varphi}$ is a $\mathrm{II}_{1}$-factor and $\mathscr{M}$ itself is also a factor.

We consider two periodic and inner homogeneous faithful normal states $\varphi$ and $\psi$ on $\mathscr{M}$. We denote by $\left\{\mathscr{M}_{n}^{\varphi}: n=0, \pm 1, \ldots\right\}$ and $\left\{\mathscr{M}_{n}^{\psi}: n=0\right.$, $\pm 1, \ldots\}$ the decompositions of $\mathscr{M}$ in [9, Theorem 11] corresponding to $\varphi$ and $\psi$ respectively. By [9, Theorem 13], $\varphi$ and $\psi$ have the same period, say $T>0$. Let $\kappa=e^{-2 \pi / T}, 0<\kappa<1$.

Following Connes' idea, we consider the tensor product $\mathscr{P}=\mathscr{M}$ $\otimes \mathscr{L}\left(\mathfrak{H}_{2}\right)$ of $\mathscr{M}$ and the $2 \times 2$-matrix algebra $\mathscr{L}\left(\mathfrak{H}_{2}\right)$. Let $\left\{e_{i, j}: i, j=1,2\right\}$ be a system of matrix units in $\mathscr{L}\left(\mathfrak{H}_{2}\right)$. Every $x \in \mathscr{P}$ is of the form

$$
x=x_{11} \otimes e_{11}+x_{12} \otimes e_{12}+x_{21} \otimes e_{21}+x_{22} \otimes e_{22},
$$

where $x_{i j} \in \mathscr{M}$. We define a faithful state $\chi$ on $\mathscr{P}$ by

$$
\chi(x)=\frac{1}{2}\left(\varphi\left(x_{11}\right)+\psi\left(x_{22}\right)\right) .
$$

Connes showed in [3] that there exists a strongly continuous one-

AMS (MOS) subject classifications (1970). Primary 46L10.

Key words and phrases. von Neumann algebras, modular automorphism group, periodic state, homogeneous state, inner homogeneous state.

${ }_{1}$ The preparation of this work was supported in part by NSF grant no. GP33696X. 
parameter family $\left\{u_{t}\right\}$ of unitaries in $\mathscr{M}$ such that $\sigma_{t}^{\chi}\left(1 \otimes e_{12}\right)=u_{t} \otimes e_{12}$ and $\sigma_{t}^{\psi}(x)=u_{t}^{*} \sigma_{t}^{\varphi}(x) u_{t}, x \in \mathscr{M}$.

LEMMA 2. $u_{s+t}=\sigma_{s}^{\varphi}\left(u_{t}\right) u_{s}{ }^{2}$

Proof. The above cocycle equality follows from the simple calculation.

$$
\begin{aligned}
u_{s+t} \otimes e_{12} & =\sigma_{s+t}^{\chi}\left(1 \otimes e_{12}\right)=\sigma_{s}^{\chi} \cdot \sigma_{t}^{\chi}\left(1 \otimes e_{12}\right) \\
& =\sigma_{s}^{\chi}\left(u_{t} \otimes e_{12}\right)=\sigma_{s}^{\chi}\left(\left(u_{t} \otimes e_{11}\right)\left(1 \otimes e_{12}\right)\right) \\
& =\left(\sigma_{s}^{\varphi}\left(u_{t}\right) \otimes e_{11}\right)\left(u_{s} \otimes e_{12}\right)=\sigma_{s}^{\varphi}\left(u_{t}\right) u_{s} \otimes e_{12} .
\end{aligned}
$$

Since $\sigma_{T}^{\varphi}=\sigma_{T}^{\psi}=\mathrm{id}, u_{T}$ must be a scalar multiple of 1 , so that one can find $1 \leqq \alpha<e^{2 \pi / T}$ with $u_{T}=\alpha^{i T} 1$. Let $v_{t}=\alpha^{-i t} u_{t}$. We have then the following properties:

$$
\begin{aligned}
v_{t}^{*} \sigma_{t}^{\varphi}(x) v_{t} & =\sigma_{t}^{\psi}(x), \quad x \in \mathscr{M} \\
v_{s+t} & =\sigma_{s}^{\varphi}\left(v_{t}\right) v_{s}=\sigma_{t}^{\varphi}\left(v_{s}\right) v_{t} \\
v_{T} & =1 .
\end{aligned}
$$

We define a one-parameter family $\left\{\rho_{t}\right\}$ of isometries of $\mathscr{M}$ onto $\mathscr{M}$ by

$$
\rho_{t}(x)=\sigma_{t}^{\varphi}(x) v_{t}, \quad x \in \mathscr{M} .
$$

From (2) and (3) we obtain the following:

$$
\begin{aligned}
\rho_{s+t} & =\rho_{s} \cdot \rho_{t} ; \\
\rho_{T} & =\mathrm{id} .
\end{aligned}
$$

For each integer $n$, let $\mathscr{V}_{n}=\left\{x \in \mathscr{M}: \rho_{t}(x)=\kappa^{i n t} x\right\}$. Since $\rho_{t}$ has period $T$, $\mathscr{V}_{n} \neq\{0\}$ for some $n$.

LeMma 3. An $x \in \mathscr{M}$ falls in $\mathscr{V}_{n}$ if and only if

$$
\sigma_{t}^{\chi}\left(x \otimes e_{12}\right)=\alpha^{i t} \kappa^{i n t}\left(x \otimes e_{12}\right) \text {. }
$$

Proof. We compute as follows:

$$
\begin{aligned}
\sigma_{t}^{\chi}\left(x \otimes e_{12}\right) & =\sigma_{t}^{\chi}\left(\left(x \otimes e_{11}\right)\left(1 \otimes e_{12}\right)\right) \\
& =\left(\sigma_{t}^{\varphi}(x) \otimes e_{11}\right)\left(u_{t} \otimes e_{12}\right)=\sigma_{t}^{\varphi}(x) u_{t} \otimes e_{12} \\
& =\alpha^{i t} \sigma_{t}(x) v_{t} \otimes e_{12}=\alpha^{i t} \rho_{t}(x) \otimes e_{12} .
\end{aligned}
$$

Thus, the assertion follows.

Therefore, we conclude that $x \in \mathscr{V}_{n}$ if and only if $\alpha \kappa^{n} \chi\left(y\left(x \otimes e_{12}\right)\right)$

\footnotetext{
${ }^{2}$ ADDED IN PROOF. This cocycle equation is mentioned in the final version of [3], which was missed from an earlier version and not available at the time when this article was finished.
} 
$=\chi\left(\left(x \otimes e_{12}\right) y\right)$ for every $y \in \mathscr{P}$.

LEMMA 4. If $x \in \mathscr{V}_{n}$ and $y \in \mathscr{M}$, then we have

$$
\alpha \kappa^{n} \psi(y x)=\varphi(x y) .
$$

Proof. For each $x \in \mathscr{V}_{n}$ and $y \in \mathscr{M}$, we have

$$
\begin{aligned}
\alpha \kappa^{n} \psi(y x) & =2 \alpha \kappa^{n} \chi\left(\left(y \otimes e_{21}\right)\left(x \otimes e_{12}\right)\right) \\
& =2 \chi\left(\left(x \otimes e_{12}\right)\left(y \otimes e_{21}\right)\right) \quad \text { by Lemma } 3 \\
& =\varphi(x y) .
\end{aligned}
$$

LEMMA 5. $\mathscr{M}_{m}^{\varphi} \mathscr{V}_{l} \mathscr{M}_{n}^{\psi} \subset \mathscr{V}_{m+l+n}$.

Proof. For each $a \in \mathscr{M}_{m}^{\phi}, b \in \mathscr{M}_{n}^{\psi}$ and $x \in \mathscr{V}_{l}$, we have

$$
\begin{aligned}
\rho_{t}(a x b) & =\sigma_{t}^{\varphi}(a x b) v_{t}=\kappa^{i m t} a \sigma_{t}^{\varphi}(x) \sigma_{t}^{\varphi}(b) v_{t} \\
& =\kappa^{i m t} a \sigma_{t}^{\varphi}(x) v_{t} v_{t}^{*} \sigma_{t}^{\varphi}(b) v_{t} \\
& =\kappa^{i m t} a \kappa^{i l t} x \kappa^{i n t} b=\kappa^{i(m+l+n) t} a x b .
\end{aligned}
$$

Since $\mathscr{M}_{m}^{\varphi}, m \geqq 1$, contains isometries and $\mathscr{M}_{n}^{\psi}, n \leqq-1$, contains co-isometries by [9], we conclude that $\mathscr{V}_{n} \neq\{0\}$ for every integer $n$. Since $\mathscr{M}_{0}^{\varphi}$ and $\mathscr{M}_{0}^{\psi}$ are both factors, we conclude that, for every pair of nonzero projections $p \in \mathscr{M}_{0}^{\varphi}$ and $q \in \mathscr{M}_{0}^{\Psi}$,

$$
p \mathscr{V}_{n} q \neq\{0\} .
$$

For an $x \in \mathscr{V}_{n}$, let $x=u h=k u$ be the right and left polar decomposition of $x$. We have then

$$
k\left(\kappa^{i n t} u\right)=\kappa^{i n t} x=\rho_{t}(x)=\sigma_{t}^{\varphi}(x) v_{t}=\sigma_{t}^{\varphi}(k) \sigma_{t}^{\varphi}(u) v_{t} .
$$

By the unicity of the polar decomposition, we get $k=\sigma_{t}^{\varphi}(k)$ and $\kappa^{\text {int }} u=\rho_{t}(u)$; hence $u \in \mathscr{V}_{n}$. Similarly, we get $h=\sigma_{t}^{\psi}(h)$.

Thus, we obtain the following:

LEMMa 6. For every $x \in \mathscr{V}_{n}$, we have

$$
x x^{*} \in \mathscr{M}_{0}^{\varphi} \text { and } x^{*} x \in \mathscr{M}_{0}^{\psi} \text {. }
$$

If $u$ is a partial isometry in $\mathscr{V}_{n}$, then we have, by (7),

$$
\varphi\left(u x u^{*}\right)=\alpha \kappa^{n} \psi\left(x u^{*} u\right), \quad x \in \mathscr{M} .
$$

Taking fact (8) into account and making use of the usual exhaustion arguments, we conclude that there exists an isometry or a co-isometry $u$ in $\mathscr{V}_{n}$ according as $\alpha \kappa^{n} \leqq 1$ or $\alpha \kappa^{n}>1$. Considering various $n$, we conclude the following: 
THEOREM 7. For periodic inner homogeneous faithful states $\varphi$ and $\psi$ on a factor $\mathscr{M}$, there exist isometries $u$ and $v$ in $\mathscr{M}$ such that

$$
\begin{aligned}
& \psi(x)=\varphi\left(u x u^{*}\right) / \varphi\left(u u^{*}\right), \\
& \varphi(x)=\psi\left(v x v^{*}\right) / \psi\left(v v^{*}\right), \quad x \in \mathscr{M} ; \\
& u u^{*} \in \mathscr{M}_{0}^{\varphi} \text { and } \quad v v^{*} \in \mathscr{M}_{0}^{\psi} .
\end{aligned}
$$

Fixing a periodic inner homogeneous state $\varphi$ on a factor $\mathscr{M}$, for each projection $p \in \mathscr{M}_{0}^{\varphi}$ we define a state $\varphi_{p}$ on $p \mathscr{M} p$ by

$$
\varphi_{p}(x)=\varphi(x) / \varphi(p), \quad x \in p \mathscr{M} p .
$$

By Theorem 7, any other periodic inner homogeneous state $\psi$ on $\mathscr{M}$ is unitarily equivalent to $\varphi_{p}$ for some $p \in \mathscr{M}_{0}^{\varphi}$; more precisely, there exists an isometry $u \in \mathscr{M}$ with $u u^{*}=p \in \mathscr{M}_{0}^{\varphi}$ such that $\psi(x)=\varphi_{p}\left(u x u^{*}\right)$ for every $x \in \mathscr{M}$. Thus, the set $\left\{\varphi_{p}: p \in \mathscr{M}_{0}^{\varphi}\right\}$ exhausts all possible periodic inner homogeneous states up to unitary equivalence.

THEOREM 8. Let $p$ and $q$ be two projections in $\mathscr{M}_{0}^{\varphi}$, and let $u$ and $v$ be isometries in $\mathscr{M}$ with $u u^{*}=p$ and $v v^{*}=q$. Let $\psi(x)=\varphi_{p}\left(u x u^{*}\right)$ and $\omega(x)=\varphi_{q}\left(v x v^{*}\right), x \in \mathscr{M}$. Then $\psi$ and $\omega$ are unitarily equivalent if and only if $\varphi(p)=\kappa^{n} \varphi(q)$ for some integer $n$.

THEOREM 9. For the state $\psi$ defined in the previous theorem, there exists $\sigma \in \operatorname{Aut}(\mathscr{M})$ with $\psi=\varphi \circ \sigma$ if and only if there exists an isomorphism $\rho$ of $\mathscr{M}_{0}^{\varphi}$ onto $p \mathscr{M}_{0}^{\varphi} p$ and a partial isometry $w$ in $\mathscr{M}_{0}^{\varphi}$ such that $\rho \cdot \theta(x)$ $=w \theta \cdot \rho(x) w^{*}, x \in \mathscr{M}_{0}^{\varphi}$, where $\theta$ denotes the isomorphism described in [9, Theorem 11].

By the following theorem, one can distinguish the algebraic type of $\mathscr{M}$ in terms of $\left\{\mathscr{M}_{0}^{\varphi}, \theta\right\}$.

THEOREM 10. Let $\mathscr{M}$ and $\mathscr{N}$ be factors equipped with periodic inner homogeneous states $\varphi$ and $\psi$ respectively. Let $\left\{\mathscr{M}_{0}, \theta\right\}$ and $\left\{\mathscr{N}_{0}, \rho\right\}$ be the relevant couples of $\mathrm{II}_{1}$-factors and isomorphisms described in [9, Theorem 11] respectively. Let $e_{-1}=\theta(1)$ and $f_{-1}=\rho(1)$. Necessary and sufficient conditions for $\mathscr{M}$ and $\mathscr{N}$ to be isomorphic are that (i) $\varphi\left(e_{-1}\right)=\psi\left(f_{-1}\right)$; (ii) there exists an isomorphism $\sigma$ of $\mathscr{N}_{0}$ onto $p \mathscr{M}_{0} p$ for some projection $p$ with $p \geqq e_{-1}$ and a partial isometry $w$ in $\mathscr{M}_{0}$ such that $w \theta \cdot \sigma(x) w^{*}=\sigma \cdot \rho(x)$, $x \in \mathscr{N}_{0}$.

Making use of the new results of Connes in [5], we can prove the following:

THEOREM 11. Let $\mathscr{M}$ be a factor equipped with a periodic homogeneous state. The existence of a periodic inner homogeneous state on $\mathscr{M}$ is equiva- 
lent to the fact that $S(\mathscr{M}) \neq\{0,1\}$, where $S(\mathscr{M})$ means the invariant of $\mathscr{M}$ defined by Connes in $[\mathbf{1}]$.

Acknowledgement. The author would like to thank Professor $\mathrm{H}$. Araki for enjoyable discussions on the subject of this paper; Professor $\mathrm{H}$. Dye for his constant encouragement; Professor J. Woods for his kind hospitality at Queen's University where this work was completed; and Dr. A. Connes for his kindness in informing the author of his new results [3] and [5], on which this work depends heavily.

\section{REFERENCES}

1. A. Connes, Un nouvel invariant pour les algèbres de von Neumann, C. R. Acad. Sci. Paris Sér. A-B 273 (1971).

2. Calcul des deux invariants d'Araki et Woods par la théorie de Tomita et Takesaki, C. R. Acad. Sci. Paris Sér. A-B 274 (1972), A175-A177.

3. - Groupe modulairé d'une algèbre de von Neumann de genre denombrable, C. R. Acad. Sci., Paris Sér. A-B 274 (1972), 1923-1926.

4. - États presque périodiques sur une algèbre de von Neumann, C. R. Acad. Sci. Paris Sér. A-B 274 (1972), A1402-A1405.

5. — A letter to the author, dated June 17, 1972

6. J. Dixmier, Les algèbres d'opérateurs dans l'espace hilbertien (Algèbres de von Neumann), Cahiers scientifiques, fasc. 25, Gauthier-Villars, Paris, 1957. MR 20 \#1234.

7. E. Størmer, Spectra of states, and asymptotically abelian $C^{*}$-algebras (to appear).

8. M. Takesaki, Tomita's theory of modular Hilbert algebras and its applications, Lecture Notes in Math., vol. 128, Springer-Verlag, Berlin and New York, 1970. MR 42 \# 5061.

9. - Periodic and homogeneous states and a von Neumann algebra. I, Bull. Amer. Math. Soc. 79 (1973), 202-206.

Department of Mathematics, University of California, Los Angeles, California 90024 